\title{
Particle acceleration in subcycle optical cells
}

\author{
F. Terranova \\ Department of Physics, University of Milano-Bicocca and INFN, Sezione di Milano-Bicocca, \\ Milano I-20126, Italy
}

(Received 16 January 2014; published 18 July 2014)

\begin{abstract}
A single laser pulse with spot size smaller than half its wavelength $\left(w_{0}<\lambda / 2\right)$ can provide a net energy gain to ultrarelativistic particles. In this paper, we discuss the properties of an optical cell consisting of $N$ subcycle pulses that propagate in the direction perpendicular to the electron motion. We show that the energy gain produced by the cell is proportional to $N$ and it is sizable even for $\mathcal{O}(1 \mathrm{TW})$ pulses.
\end{abstract}

DOI: 10.1103/PhysRevSTAB.17.071301

PACS numbers: $41.75 . J v$

Modern conventional accelerators transfer only a small amount of energy to particles per unit cell. Large energy gradients are achieved combining the cells in periodic structures and phase locking the particle motion to the rf cavities. On the other hand, phase slippage is difficult to be avoided in laser-based accelerators and acceleration is achieved by a few beam-pulse interactions but, in this case, the energy transfer per pulse can be very large due to the huge electric fields available in lasers. Unlike rf-based accelerators, the interaction time $\tau$ of the particle with the laser is much longer that the period $T$ of the laser fields. Such a long interaction time causes several drawbacks. Plasma-based accelerators [1] tend to be prone to instabilities when $\tau \gg T$. This difficulty has been overcome by the development of high-power sub-ps lasers and resulted into large energy gains and beams of unprecedented quality [2]. Similarly, laser accelerators in vacuum [3,4] require special geometrical configurations of the laser pulse to bypass the limitations imposed by the Lawson-Woodward theorem [5] and reach a finite energy gain even if $\tau \gg T$ [6]. These challenges have not been overcome yet, and vacuum-based techniques are much less developed than laser-plasma accelerators. As discussed in this paper, however, the impressive progresses in subcycle laser technology achieved in the past decade could boost vacuum acceleration in a way similar to what sub-ps lasers have done in the past for plasma-based accelerators.

Attosecond optics allows for the focusing of pulses to spot sizes significantly smaller than $\lambda$ [7], and for creation $[8,9]$, manipulation and regeneration of isolated subcycle pulses [10]. In addition, accelerators based on subcycle pulses can be operated in the $\tau<T$ regime: particles that are injected nearly perpendicularly with respect to the pulse propagation axis (see Fig. 1) will experience only a fraction of the oscillating electric field and, if properly phased, they

Published by the American Physical Society under the terms of the Creative Commons Attribution 3.0 License. Further distribution of this work must maintain attribution to the author(s) and the published article's title, journal citation, and DOI. will acquire a net energy gain when they cross the focus of the pulse. A set of $N$ subcycle pulses can be employed to transfer a fixed amount of energy to the particles through $N$ beam-pulse interactions. If the overall energy gain is constant and the beam is stable in phase space, then the $N$-pulse optical lattice acts as a single accelerating unit (optical cell) with a well defined transfer function. Particles crossing an optical cell will experience an energy gain that, in general, will depend on the initial position of the particle; moreover, the particles will be deflected in the transverse plane ( $y-z$ in Fig. 1) by the longitudinal electric field $E_{z}$ and by the ponderomotive force. In this paper we study the accelerating properties of the optical cell and show that, in suitable conditions, this device can provide an energy gain proportional to $N$ and is described by a transfer function similar to the one of a defocusing lens.

The advantage of an optical cell compared to standard rf cells resides mostly in the intensity of the electric field, which can exceed the rf field by more than 6 orders of magnitude. In addition, the size of the cell in the $x$ direction is $\mathcal{O}(N \lambda)$ and, therefore, is suitable for tabletop

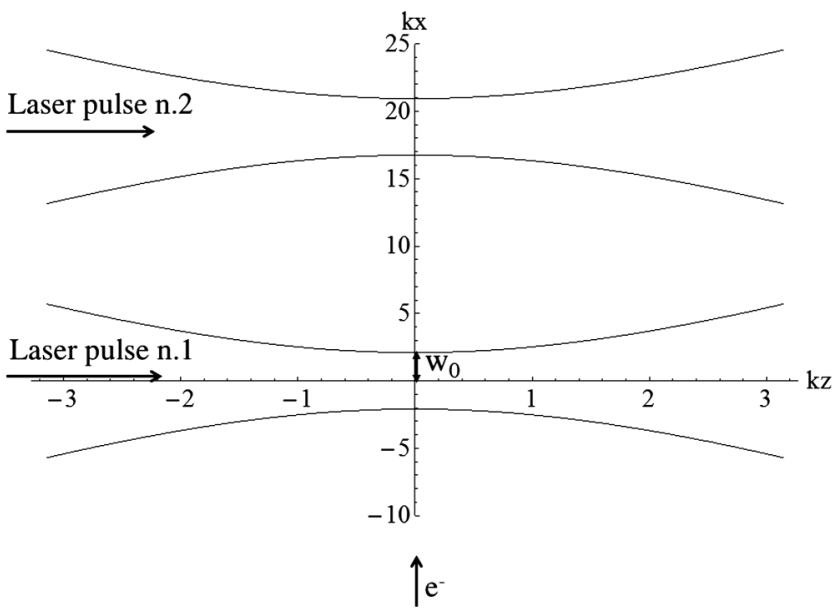

FIG. 1. A $N=2$ optical cell. The laser pulses propagate along the $z$ axis and the particles are injected along $x$. Distances are expressed in units of $\lambda: k z$ (or $k x)=2 \pi$ corresponds to a shift of $\lambda$ in the $z(x)$ direction. $w_{0}$ is the beam spot (see text). 
acceleration. On the other hand, the acceptance of the optical cell, i.e., the region in the transverse plane where particles can experience acceleration, is small $(\mathcal{O}(\lambda) \sim 1-10 \mu \mathrm{m})$ and comparable to the vertical beam size of high energy accelerators or free-electron lasers [11] for optical or IR lasers.

In this paper, we consider an optical cell in its simplest configuration (Fig. 1): a set of $N$ pulses reflected back and forth along the $z$ axis and focused to an area $<\lambda^{2}$ at $z=z_{0}$ $\left(z_{0}=0\right.$ in Fig. 1). The length of the pulse along $z$ is assumed to be $\gg \lambda$ so that the light beam has a stationary focus at $z=z_{0}$. The pulse profile at $z$ is circular with radius $w(z)=w_{0} \sqrt{1+\left(\frac{z-z_{0}}{z_{R}}\right)^{2}}, z_{R}=k w_{0}^{2} / 2$ being the Rayleigh length of the pulse and $k=2 \pi / \lambda$ the wave number of the light at central frequency. The electromagnetic fields in the proximity of the focus can be derived following the approach of Davis and McDonald [12,13]. The light beam is modeled employing a vector potential A linearly polarized along $x$ and expressing $\mathbf{A}$ as a perturbative expansion of the diffraction angle $\epsilon=w_{0} / z_{R}$. Such treatment, which is consistent as long as $\epsilon<1\left(w_{0}>\lambda / \pi\right)$, provides the electric and magnetic fields in the proximity of the focus as a function of the transformed variables:

$$
\begin{aligned}
\xi & =x / x_{0}, \quad v=y / w_{0}, \quad \zeta=z / z_{R}, \\
r^{2} & =x^{2}+y^{2}, \quad \rho=r / w_{0}
\end{aligned}
$$

and of the plane wave phase $\eta=\omega t-k z$. At next-toleading order [14] the fields are

$$
\begin{gathered}
E_{x}=E_{0} \frac{w_{0}}{w} e^{-\frac{r^{2}}{w^{2}}}\left\{S_{0}+\epsilon^{2}\left[\xi^{2} S_{2}-\frac{\rho^{4} S_{3}}{4}\right]+\mathcal{O}\left(\epsilon^{4}\right)\right\} \\
E_{y}=E_{0} \frac{w_{0}}{w} e^{-\frac{r^{2}}{w^{2}}} \xi v\left\{\epsilon^{2} S_{2}+\mathcal{O}\left(\epsilon^{4}\right)\right\} \\
E_{z}=E_{0} \frac{w_{0}}{w} e^{-\frac{r^{2}}{w^{2}}} \xi\left\{\epsilon C_{1}+\epsilon^{3}\left[-\frac{C_{2}}{2}+\rho^{2} C_{3}-\frac{\rho^{4} C_{4}}{4}\right]+\mathcal{O}\left(\epsilon^{5}\right)\right\} \\
B_{y}=E_{0} \frac{w_{0}}{w} e^{-\frac{r^{2}}{w^{2}}}\left\{\epsilon^{2}\left[\frac{\rho^{2} S_{2}}{2}-\frac{\rho^{4} S_{3}}{4}\right]+\mathcal{O}\left(\epsilon^{4}\right)\right\} \\
B_{z}=E_{0} \frac{w_{0}}{w} e^{-\frac{r^{2}}{w^{2}}} v\left\{\epsilon C_{1}+\epsilon^{3}\left[\frac{C_{2}}{2}+\frac{\rho^{2} C_{3}}{2}-\frac{\rho^{4} C_{4}}{4}\right]+\mathcal{O}\left(\epsilon^{4}\right)\right\}
\end{gathered}
$$

where

$$
S_{n}=\left(\frac{w_{0}}{w}\right)^{n} \sin \left(\psi+n \psi_{G}\right), \quad C_{n}=\left(\frac{w_{0}}{w}\right)^{n} \cos \left(\psi+n \psi_{G}\right) .
$$

The phases entering Eq. (8) are the ones describing standard Gaussian beams [15]: the plane wave phase $\eta$, the Gouy phase $\psi_{G} \equiv \tan ^{-1} \zeta$, the initial phase $\psi_{0}$, and the phase advance due to the curvature of the wave front:

$$
\psi_{R}=\frac{k r^{2}}{2\left(z-z_{0}+\frac{z_{R}^{2}}{z-z_{0}}\right)} .
$$

The overall phase $\psi$ is $\psi=\psi_{0}+\eta-\psi_{R}+\psi_{G}$. The fields have been computed analytically up to $\epsilon^{11}$ in Ref. [16]. In the study of the optical cell described below we retain all terms up to $\epsilon^{5}$.

A single pulse $(N=1)$ optical cell can be operated even in the $\tau>T$ regime [4,17] but in this case the particle must be injected at an angle close to the $z$ direction. For $\tau>T$ acceleration is mostly driven by $E_{z}$ and the ponderomotive force. Employing Petawatt class lasers [18] the energy gain can be very large although the acceleration regime is highly nonlinear [19,20]. For $\tau<T$ [21], the particle of charge $q$ injected in the proximity of the $x$ axis experiences only a portion of the $E_{x}$ period and net acceleration is caused mostly by the $q E_{x}$ linear term. The $B_{y}$ and $E_{z}$ fields contribute to the deflection angle in the $x-z$ plane. This can be demonstrated solving numerically the equation of motion of the particle (electron, $q \equiv-e$ ) in the electric fields of Eqs. (2)-(7):

$$
\frac{d \mathbf{p}}{d t}=-e[\mathbf{E}+c \boldsymbol{\beta} \times \mathbf{B}], \quad \frac{d E}{d t}=-e c \boldsymbol{\beta} \cdot \mathbf{E} .
$$

Numerical integration of Eq. (10) is eased if the motion is described in units of $T$ and the fields are expressed as $\tilde{\mathbf{E}} \equiv(e / m c \omega) \mathbf{E}$ and $\tilde{\mathbf{B}} \equiv(e / m \omega) \mathbf{B}$. The resulting equation [19] is

$$
\frac{d \boldsymbol{\beta}}{d \omega t}=\frac{1}{\gamma}[\boldsymbol{\beta}(\boldsymbol{\beta} \cdot \tilde{\mathbf{E}})-(\tilde{\mathbf{E}}+\boldsymbol{\beta} \times \tilde{\mathbf{B}})] .
$$

In these formulas, $-e$ and $m$ are the charge and electron mass in SI units, $\boldsymbol{\beta}$ is the electron velocity normalized to $c$ and $\gamma=\left(1-\beta^{2}\right)^{-1 / 2}$. Figure 2 shows the net energy gain $\Delta E=\left(\gamma-\gamma_{0}\right) m c^{2}$ for a $100 \mathrm{MeV}$ electron $\left(\gamma_{0}=195.7\right)$ injected along the $x$ axis as a function of $w_{0}$. The initial phase $\psi_{0}$ is chosen in order to have the electron at the center of the focus when $\left|E_{x}(\omega t)\right|$ reaches its maximum. The laser pulse considered corresponds to $P_{0}=1 \mathrm{TW}$ and $10 \mathrm{TW}$ power Gaussian beams focused to a beam spot $w_{0}$. In Fig. 2, $w_{0}$ ranges from $w_{0}=\lambda / \pi$ to $\lambda / 2$. The power crossing the disk of area $\pi w_{0}^{2}$ at $z_{0}=0$ is $P_{0}\left(1-e^{-2}\right) \simeq 0.865 P_{0}$. The laser intensity $I_{0}$ at $z=z_{0}$ and $r^{2}=0$ is proportional to the overall beam power: $P_{0}=\pi w_{0}^{2} I_{0} / 2$. The electric field is

$$
E_{0}=\frac{1}{w_{0}} \sqrt{\frac{4 P_{0}}{\pi \epsilon_{0} c}} .
$$




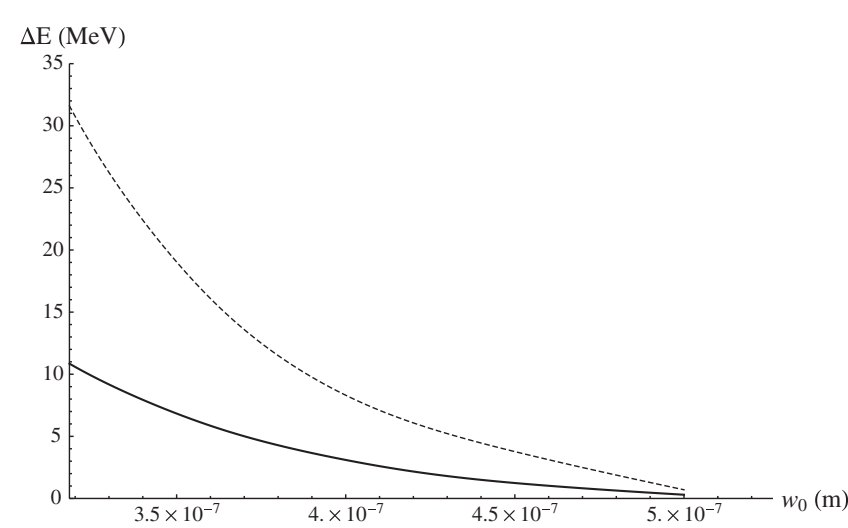

FIG. 2. Net energy gain $\Delta E$ in $\mathrm{MeV}$ versus $w_{0}$ for a $100 \mathrm{MeV}$ electron injected into the focus of a 1 TW (black continuous line) and $10 \mathrm{TW}$ (dashed line) Gaussian beam $(\lambda=1 \mu \mathrm{m})$. The smallest value $w_{0}=\lambda / \pi$ corresponds to the limit of the perturbative expansion of the fields $(\epsilon=1)$. The largest value corresponds to $w_{0}=\lambda / 2$.

For $w_{0}=\lambda / 3$ and $P_{0}=1 \mathrm{TW}, I_{0}$ is $5.7 \times 10^{20} \mathrm{~W} / \mathrm{cm}^{2}$ and $E_{0}=6.6 \times 10^{13} \mathrm{~V} / \mathrm{m}$.

As expected, a net energy gain due to the electric field $E_{x}$ is visible as long as the particle experiences just a fraction of the oscillation period. In particular, no energy gain is observed when $w_{0}>\lambda / 2$ because the interaction time for an ultrarelativistic particle $\tau \simeq 2 w_{0} / c$ is larger than an optical cycle and hence, the electron experiences both an accelerating and a decelerating electric field with nearly equal strength. For $w_{0}<\lambda / 2$ the energy gain $\Delta E$ grows linearly with $E_{0}$ and is proportional to $\sqrt{P_{0}}$. This scaling is in agreement with results obtained under paraxial conditions [22] and in nonparaxial treatments [23,24]. It is worth mentioning that an increase of the laser wavelength at fixed $P_{0}$ does not increase the energy gain per pulse for ultrarelativistic particles: since $E_{0} \sim w_{0}^{-1} \sim 1 / \lambda$ and the interaction time $\tau$ is $\sim 2 w_{0} / c$, the momentum change experienced during the pulse-electron interaction is $-e \int_{0}^{\tau} E_{x} d t \simeq-e \tau E_{0}$ and it is thus independent of $\lambda$. Still, the exploitation of mid-IR lasers $(\lambda \simeq 10 \mu \mathrm{m})$, as $\mathrm{CO}_{2}$ lasers [25], would be particularly rewarding in this scheme because it would increase significantly the acceptance of the cell. Progress toward sub-ps, 10 TW power $\mathrm{CO}_{2}$ lasers are very encouraging [26] but the capability to reach the $w_{0}<\lambda / 2$ condition in these lasers has not been demonstrated, yet.

The electron motion in the $z$ direction is determined by the interplay between $B_{y}$ and $E_{z}$. In the proximity of the focus the former boosts the electron toward the direction of motion of the laser pulse, while for $z \rightarrow z_{0}$ and $r \rightarrow 0, E_{z}$ is $\simeq 0$ and the effect of the longitudinal field $E_{z}$ is marginal. Far from the focus, $B_{y}$ decreases steeply and the contribution of $E_{z}$ becomes sizable. Figure 3 shows the net energy gain $\Delta E$ for a $100 \mathrm{MeV}$ electron injected along $x$ at $z_{0}=0$ and crossing three pulses $\left(P_{0}=1 \mathrm{TW}, w_{0}=\lambda / 3\right)$

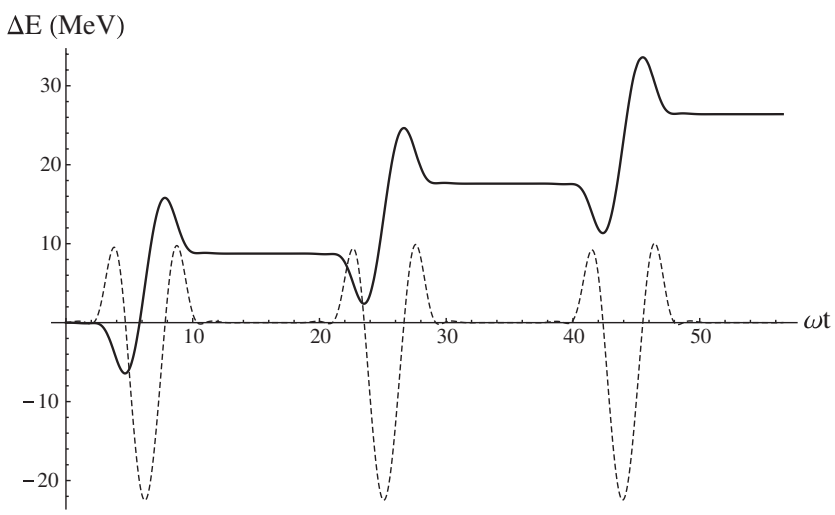

FIG. 3. Black line: net energy gain $\Delta E$ in $\mathrm{MeV}$ versus $\omega t$ for a $100 \mathrm{MeV}$ electron injected into the focus of three 1 TW Gaussian beams $\left(\lambda=1 \mu \mathrm{m}, w_{0}=\lambda / 3\right)$. The accelerating field $\tilde{E}_{x}$ experienced by the particle at the time $\omega t$ is also shown (dashed line).

located at a distance $3 \lambda$ in $x(\Delta k x=6 \pi)$. The accelerating field $\tilde{E}_{x}$ experienced along its trajectory is also shown.

The transverse motion of the particle is described in Fig. 4. The continuous and short-dashed lines indicate the position and $\beta_{z}$ of the particle along $k z(k z=2 \pi$ corresponds to $z=\lambda$ ) as a function of $\omega t$. The long-dashed and dotted lines show the values of the fields $\tilde{B}_{y}$ and $\tilde{E}_{z}$ (divided by 30 to ease the reading of the plot) experienced along the trajectory. For an ultrarelativistic particle $(100 \mathrm{MeV}$ electron in Fig. 4) starting at $k x_{0}=-2 \pi$, the focus is reached at $\omega t=2 \pi$. Far from the focus, both $E_{z}$ and $B_{y}$ are positive and deflect the particle toward negative values of $z$. Near the focus, $B_{y}$ is large and negative and accelerates the electron along positive $z$. The net result is that the two effects partially cancel when the electron crosses the laser beam, and the overall deflection is much smaller than one could envisage from the $\boldsymbol{\beta} \times \mathbf{B}$ force only. This cancellation effect has been noted in the 1990s and it is known to severely limit the energy transfer along $z$ in the $\tau>T$

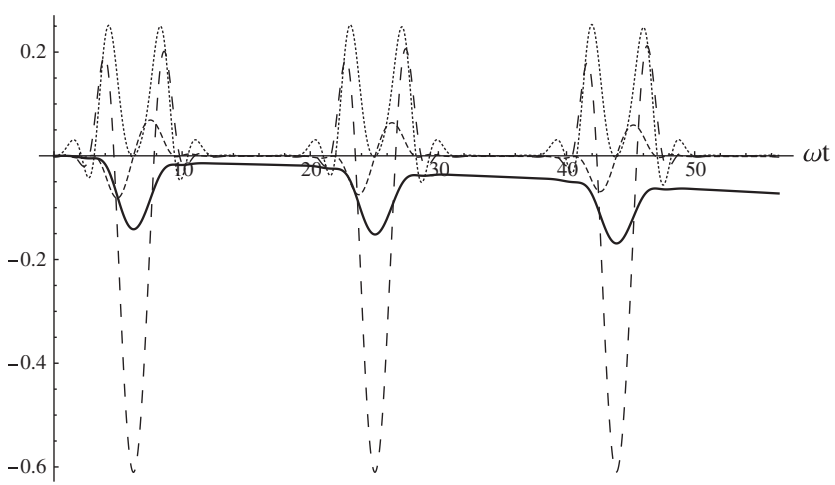

FIG. 4. Position of the particle along $k z$ (black continuous line; $k z=2 \pi$ corresponds to $z=\lambda$ ) and $\beta_{z}$ of the particle (shortdashed line) as a function of $\omega t$. Beam and laser parameters are the same as for Fig. 3. The long-dashed and dotted lines show the values of $\tilde{B}_{y} / 30$ and $\tilde{E}_{z} / 30$ experienced along the trajectory. 
regime [4,27]. If $\tau<T$, however, the acceleration is due to $E_{x}$ and the cancellation above helps to stabilize the particles in the transverse plane. It is also worth mentioning that the energy gain and the deflection of the electron is correlated with the relative position and angle between the injected electron and the laser. Besides acceleration, this feature might be exploited for ultrafast diagnostics of subcycle laser pulses [28].

In an optical cell of $N$ pulses, the final energy reached by the particle and its trajectory in the transverse plane depends on the initial position of the particle itself. If particles are ultrarelativistic, they will always arrive in phase with the next pulse, except for the slippage in $k x$ due to the fact that $\beta_{z} \neq 0$ and the trajectory is no more rectilinear. The small distance among the pulses and the cancellation effect of $B_{y}$ and $E_{z}$ in the transverse plane make the phase slippage quite small and the transfer function of the cell very regular. This is demonstrated for $P_{0}=1 \mathrm{TW}, w_{0}=\lambda / 3$ pulses and $100 \mathrm{MeV}$ electrons in Figs. 5 and 6. Figure 5 shows the energy gain as a function of $N$ for particles injected at different $\Delta \equiv k z(t=0)-k z_{0}$ (in the plot, $\Delta$ ranges between $-\pi / 3$ and $\pi / 3$ ). Note that the

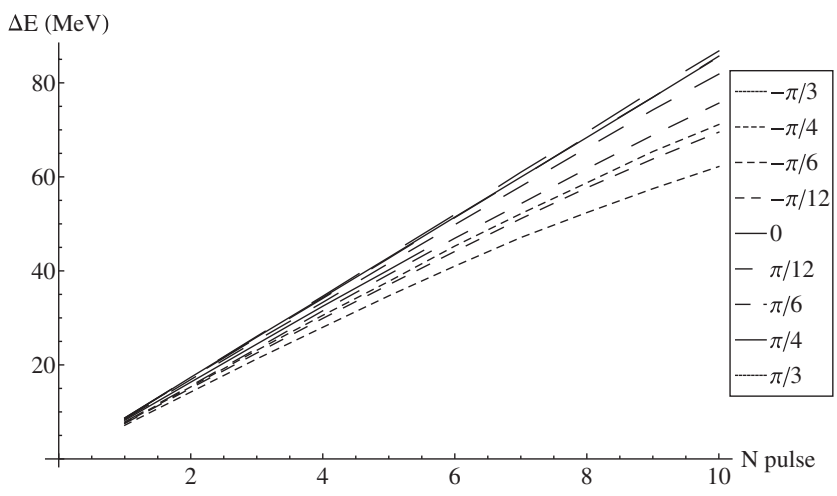

FIG. 5. Energy gain $\Delta E$ (in $\mathrm{MeV}$ ) after crossing $N$ laser pulses for $100 \mathrm{MeV}$ electrons injected along $x$ at different positions in the $z$ axis.

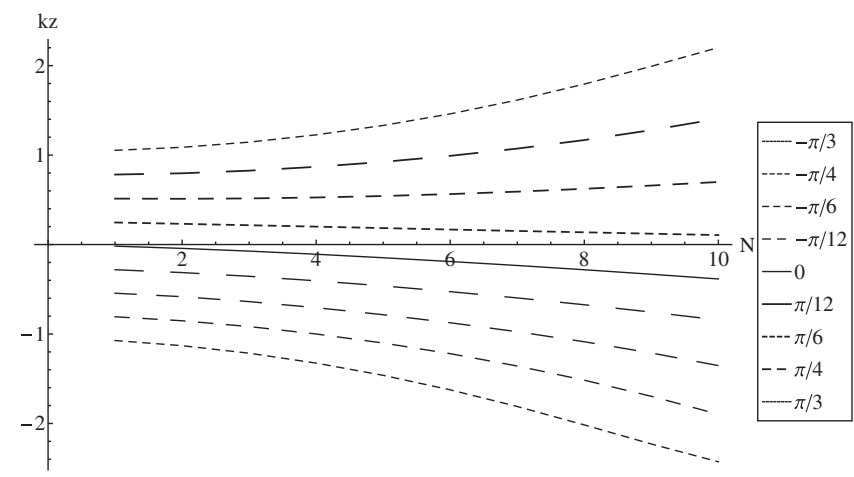

FIG. 6. $k z$ position of the particles after crossing $N$ laser pulses for $100 \mathrm{MeV}$ electrons injected along $x$ at different positions in the $z$ axis. maximum gain is observed for electrons that are injected with a slight offset with respect to $z_{0}(\Delta \simeq \pi / 12)$. This is due to the residual bending in the transverse plane experienced by particle injected directly at the focus $(\Delta=0$, see Fig. 4). It is also visible in Fig. 6, where the $\mathrm{kz}$ position of the particle after $N$ electron-pulse interactions is shown. The energy spread at $N=10$ is $13 \%$ and the maximum transverse distance is comparable to the $\mathcal{O}(\lambda)$ size of the pulse in the $z$ direction. Particles that are injected far from the focus will experience a negligible acceleration and will flow unperturbed, while in-focus electrons will experience acceleration (Fig. 5) and defocusing (Fig. 6). The superposition of this nonaccelerated electron halo with in-focus electrons is a source of nonlaminar flow. To reduce the halo, it is therefore important that the injected beam has a transverse size comparable with the laser beam spot $w_{0}$. The finite contrast of the laser pulses, i.e., the presence of pedestals along $z$, does not affect significantly the behavior of the cell since pedestals do not overlap spatially with the accelerated electron beam. Similarly, deviation from Gaussianity of the pulse envelope can affect the net energy gain per pulse $\Delta E$ through the $-e \int_{0}^{\tau} E_{x} d t \simeq-e \tau E_{0}$ integral. In this case, $\Delta E$ remains sizable if the $\tau<\lambda / c$ condition is fulfilled.

The results of Figs. 4-6 are drawn assuming ideal phase synchronization among the pulses and between pulses and the incoming electrons. A systematic desynchronization of the pulses with respect to the injected electrons ("walk") reduces the overall gain and shifts the transfer function of Fig. 6. For instance, a $1 \mathrm{fs}$ walk will reduce the energy gain by about $25 \%$. Random pulse-to-pulse phase variations that preserve the $\tau<\lambda / c$ condition (e.g., a 1 fs "jitter" among pulses) average out and will have no sizable effects in the operation of the cell. In fact, synchronization and carrier-envelope [29] phase control of femtosecond pulses at the level that is requested for this application are needed in other research fields too, and have been achieved by attosecond pulse generators [30]. Such a level of stability control in laser pulses, however, has never been demonstrated for the particular configuration of the optical cell, where sub-fs synchronization and the $w_{0}<\lambda / 2$ condition have to be fulfilled for all pulses confined within the cell. Similarly, beam-laser synchronization techniques developed for free-electron-lasers (FEL) and external-injection laser-wakefield acceleration [31] are aimed to a precision of $\mathcal{O}(10)$ fs. Those beams can be employed for a proof of principle of this acceleration scheme but the level of synchronization is still significantly larger than the halfperiod scale (1.7 fs for $\lambda=1 \mu \mathrm{m})$ needed for a fully efficient exploitation of the optical cell. For the beam parameters of [31], the ratio $(R)$ between the energy transferred to the particles through the cell and the total laser pulse energy stored in the cell is $R<0.1$. In this regime the effects of laser depletion, distortion, and space charge can be neglected, while the study of the high beam 
loading regime $(R \simeq 1)$ requires a full multiparticle simulation. Similarly, a multiparticle simulation of the whole system of optical cells and refocusing units would be needed to study the occurrence of beam breakup instabilities. Still, the small size of the cell and the absence of plasma sheaths make this scheme less sensitive to such class of instabilities and to the resonant growth of head-tail oscillations inside the optical cell.

Figure 6 shows that the cell defocuses the electrons in the $x-z$ plane. It is, thus, inconvenient to build optical cells with $N \gg 10$ before proceeding to particle refocusing because peripheral particles will not experience large values of $E_{x}$ at the end of the cell. Note also that conventional refocusing units have lengths of $\mathcal{O}(1 \mathrm{~m})$. The minimum distance between optical cells is thus much larger than the longitudinal size of the cell. The overall energy gain of an optical cell is independent of the initial energy of the electron as far as the particle is ultrarelativistic but, as for standard lenses, the cell causes chromatic aberration. For a $1 \mathrm{GeV}$ initial energy electron the $N_{0}=10$ cell accelerates particles up to $\Delta E \simeq 80 \mathrm{MeV}$ but, in this case, the spread in the transverse plane is $\sim 10$ times smaller because the magnetic rigidity is proportional to the particle momentum. At larger momenta and ultrarelativistic particles $(E \simeq p c)$, it is thus appropriate to employ cells with $N \simeq N_{0} \cdot p(\mathrm{MeV}) / 100$.

Particles that are injected close to the $y=0$ axis at any position in $z$ will remain in the $x$ - $z$ plane. On the other hand, if they are significantly displaced in $y(\Delta k y= \pm \pi / 3$ in Fig. 7) they will experience the fields near pulse
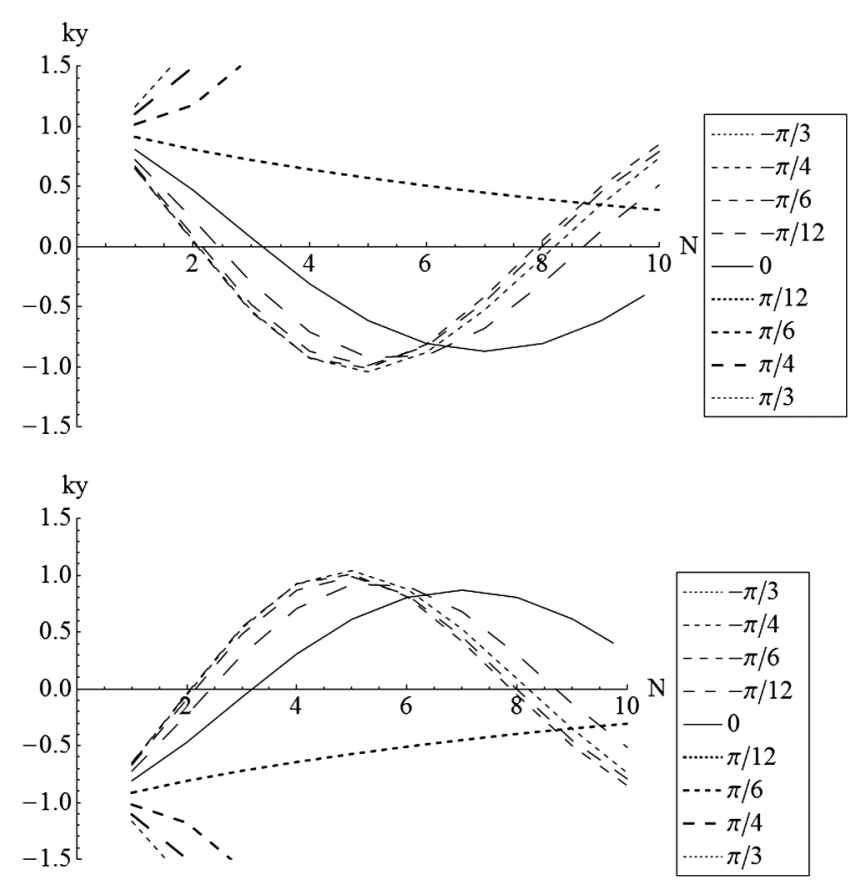

FIG. 7. $k y$ position of the particles after crossing $N$ laser pulses for $100 \mathrm{MeV}$ electrons injected along $x$ at different positions in the $z$ axis. The electrons are injected with an offset in $y$ equal to $\pi / 3$ (top plot) and to $-\pi / 3$ (bottom plot). boundaries. In general, these particles will oscillate around the $y=0$ axis, but electrons that are also displaced in the $k z>0$ direction will be driven outside the cell acceptance. This is shown in Fig. 7 where the $k y$ position of the particles is traced as a function of the number of crossed laser pulses. Figure 7 refers to $100 \mathrm{MeV}$ electrons injected along $x$ at different positions in the $z$ axis with an offset $\Delta k y$ in the $y$ direction equal to $\pi / 3$ (top plot) and to $-\pi / 3$ (bottom plot).

In conclusion, even the simplest configuration of the optical cell can be operated with ultrarelativistic particles in order to increase the energy to an amount proportional to $N$. The energy gain is sizable if the spot size is smaller than $\lambda / 2$ and scales as $P_{0}^{1 / 2}$. The optical cell can be operated with $N \simeq 10$ for $100 \mathrm{MeV}$ electrons and the optimum number of pulses increases linearly with the initial momentum of the particles. The acceptance of the optical cell, i.e., the region in the transverse plane where particles can experience acceleration, is of the order of $w_{0}$. From a practical point of view, it represents the most notable limitation of this scheme: stable and efficient acceleration of particles requires injected beams of $\mu \mathrm{m}$ size for nearinfrared lasers and beam-laser synchronization at the sub-fs level. As mentioned above, beam manipulation techniques developed for FEL allow for a proof of principle of the optical cell concept but high transport and acceleration efficiency through optical cells remains a major technological challenge.

The author gratefully acknowledges comments and suggestions from S.V. Bulanov, R. Felici, K. T. McDonald, and F. Pegoraro.

[1] E. Esarey, C. B. Schroeder, and W. P. Leemans, Rev. Mod. Phys. 81, 1229 (2009).

[2] See e.g., S. Banerjee, S. Y. Kalmykov, N. D. Powers, G. Golovin, V. Ramanathan, N. J. Cunningham, K. J. Brown, S. Chen et al., Phys. Rev. ST Accel. Beams 16, 031302 (2013), and references therein.

[3] E. D. Courant, C. Pellegrini, and W. Zakowicz, Phys. Rev. A 32, 2813 (1985); H. Hora, Nature (London) 333, 337 (1988); T. Plettner, R. L. Byer, E. Colby, B. Cowan, C. M. S. Sears, J. E. Spencer, and R. H. Siemann, Phys. Rev. Lett. 95, 134801 (2005); P.-L. Fortin, M. Piché, and C. Varin, J. Phys. B 43, 025401 (2010).

[4] E. Esarey, P. Sprangle, and J. Krall, Phys. Rev. E 52, 5443 (1995).

[5] J. D. Lawson, IEEE Trans. Nucl. Sci. 26, 4217 (1979); M. Woodward, J. Inst. Electr. Eng. 93, 1554 (1947).

[6] L. Shao, D. Cline, X. Ding, Y. K. Ho, Q. Kong, J. J. Xu, I. Pogorelsky, V. Yakimenko, and K. Kusche, Nucl. Instrum. Methods Phys. Res., Sect. A 701, 25 (2013).

[7] R. Dorn, S. Quabis, and G. Leuchs, Phys. Rev. Lett. 91, 233901 (2003).

[8] E. Goulielmakis, M. Schultze, M. Hofstetter et al., Science 320, 1614 (2008). 
[9] G. Krauss, S. Lohss, T. Hanke, A. Sell, S. Eggert, R. Huber, and A. Leitenstorfer, Nat. Photonics 4, 33 (2010).

[10] Z. Chang, Fundamentals of Attosecond Optics (CRC Press, Boca Raton, FL, 2011).

[11] J. Beringer et al. (Particle Data Group), Phys. Rev. D 86, 010001 (2012).

[12] L. W. Davis, Phys. Rev. A 19, 1177 (1979).

[13] K. T. McDonald (unpublished), available at http://www .hep.princeton.edu/ mcdonald/accel.

[14] Y. I. Salamin, G. R. Mocken, and C. H. Keitel, Phys. Rev. ST Accel. Beams 5, 101301 (2002).

[15] A. E. Siegman, Lasers (University Science Books, Sausalito, CA, 1986).

[16] Y. I. Salamin, Appl. Phys. B 86, 319 (2007).

[17] P. X. Wang, Y. K. Ho, X. Q. Yuan, Q. Kong, N. Cao, A. M. Sessler, E. Esarey, and Y. Nishida, Appl. Phys. Lett. 78, 2253 (2001); K. P. Singh, Appl. Phys. Lett. 87, 254102 (2005); P. X. Wang, J. F. Hua, Y.Z. Lin, and Y. K. Ho, Phys. Lett. A 300, 76 (2002).

[18] G. A. Mourou, T. Tajima, and S. V. Bulanov, Rev. Mod. Phys. 78, 309 (2006).

[19] Y. I. Salamin and C. H. Keitel, Phys. Rev. Lett. 88, 095005 (2002); Y. I. Salamin, Phys. Rev. A 73, 043402 (2006).

[20] J. L. Hsu, T. C. Katsouleas, W. B. Mori, C. B. Schroeder, and J. S. Wurtele, AIP Conf. Proc. 396, 21 (1997).
[21] H. M. Lai, Phys. Fluids 23, 2373 (1980).

[22] L. J. Wong and F. X. Kärtner, Appl. Phys. Lett. 99, 211101 (2011).

[23] V. Marceau, A. April, and M. Piché, Opt. Lett. 37, 2442 (2012).

[24] S. M. Sepke and D. P. Umstadter, J. Opt. Soc. Am. B 23, 2295 (2006), and references therein.

[25] Z. Harman, Y. I. Salamin, B. J. Galow, and C. H. Keitel, Phys. Rev. A 84, 053814 (2011).

[26] I. V. Pogorelsky, V. Yakimenko, M. Polyanskiy, P. Shkolnikov, M. Ispiryan, D. Neely, P. McKenna, D. Carroll, Z. Najmudin, and L. Willingale, Nucl. Instrum. Methods Phys. Res., Sect. A 620, 67 (2010).

[27] P. Sprangle, E. Esarey, J. Krall, and A. Ting, Opt. Commun. 124, 69 (1996); K. T. McDonald, Phys. Rev. Lett. 80, 1350 (1998).

[28] We are grateful to the anonymous referee for bringing this issue to our attention.

[29] E. Moon, H. Wang, S. Gilbertson, H. Mashiko, M. Chini, and Z. Chang, Laser Photonics Rev. 4, 160 (2010).

[30] See e.g., Chap. 3 of [10] and references therein.

[31] A. R. Rossi, A. Bacci, M. Belleveglia, E. Chiadroni, A. Cianchi, G. Di Pirro, M. Ferrario, A. Gallo et al., Nucl. Instrum. Methods Phys. Res., Sect. A 740, 60 (2014). 